\title{
TEXTURE DISCRIMINATION USING WAVELETS
}

\author{
Michael Unser \\ Biomedical Engineering and Instrumentation Program, NCRR, Bldg. 13, Room 3W13 \\ National Institutes of Health, Bethesda, MD 20892 USA
}

\begin{abstract}
A new approach to the characterization of texture properties at multiple scales using an overcomplete wavelet transform is described. It is shown that this representation constitutes a tight frame of $l_{2}$ and that it has a fast iterative algorithm. A texture is characterized by a set of channel variances estimated at the output of the corresponding filterbank. Classification experiments with 12 Brodatz textures indicate that the discrete wavelet frame (DWF) approach is superior to a standard (critically sampled) wavelet transform feature extraction. This result also suggests that this approach should perform better than most traditional single resolution techniques (co-occurrences, local linear transform, etc...). A detailed comparison of the classification performance of various orthogonal and biorthogonal wavelet transforms is also provided. Finally, the DWF feature extraction technique is incorporated into a simple multi-component texture segmentation algorithm and some illustrative examples are presented.
\end{abstract}

\section{INTRODUCTION}

Most traditional statistical approaches to texture are restricted to the analysis of spatial interactions over relatively small neighborhoods. As a consequence, their performance is best for the class of so-called micro-textures. One way to overcome the intrinsic limitations of a single-scale analysis is to use the discrete wavelet transform (DWT), which provides a precise and unifying framework for the characterization of a signal at multiple resolution [1]. Besides the pioneening work of Mallat on texture analysis [1], there have now been several studies on texture classification with particular attention to the use of wavelet packets $[2,3]$, which constitute a multiband extension of the pyramid-structured wavelet transform.

This paper introduces the use of an overcomplete wavelet decomposition (discrete wavelet frame - DWF) in which the output of the filter banks are not subsampled. Unlike other wavelet-based approaches, this should result in a texture description invariant with respect to translations of the input signal. The main aspects of this wavelet representation that are investigated in this work are as follows:

- The study of the properties of the DWF : energy conservation, fast algorithm, and the link with the mathematical concept of frame.

- The experimental comparison of the performance of various feature extraction techniques for the classification of 12 Brodatz textures. The comparison includes standard single resolution techniques, as well as biorthogonal wavelet transforms (Bsplines and D-splines) that are very similar to a hierarchical Gabor transform [4].

- The extension of this approach for texture segmentation. Morevover, it will be shown that a single scale DWF feature extraction is in fact equivalent to an analysis by local linear transform $[5,6]$. One of the benefits of this connection is that this latter method is well understood; it also compares favorably with most traditional approaches to texture and can therefore be considered as the reference method for single scale analysis.

\section{DISCRETE WAVELET FRAMES}

2.1 Filter bank specification and properties

The wavelet representations considered here are entirely specified in terms of a prototype lowpass filter $h$ that satisfies the standard quadrature mirror filter condition:

$$
H(z) H\left(z^{-1}\right)+H(-z) H\left(-z^{-1}\right)=1 \text {, }
$$

where $H(z)$ denotes the $z$-transform of $h$. The complementary highpass filter $g$ is obtained by shift and modulation

$$
G(z)=z H\left(-z^{-1}\right) .
$$

The wavelet filterbank is then defined iteratively in the $z$ transform domain using the scale index $i$ :

$$
\left\{\begin{array}{l}
H_{i+1}(z)=H\left(z^{z^{2}}\right) H_{i}(z) \\
G_{i+1}(z)=G\left(z^{z^{r}}\right) H_{i}(z)
\end{array}, \quad(i=0, \ldots, I-1)\right.
$$

with the initial condition $H_{0}(z)=1$. It is not difficult to verify that these filters satisfy the identity

$$
\left|H_{i}\left(e^{j 2 \pi f}\right)\right|^{2}+\sum_{j=1}^{j}\left|G_{j}\left(e^{j 2 \pi f}\right)\right|^{2}=1,
$$

and therefore provide a full coverage of the frequency domain. Because of its intrinsic multiresolution structure, such a filter bank can be implemented very efficiently using the procedure summarized in Fig. 1. Note that the complexity of this algorithm is the same for all iterations.

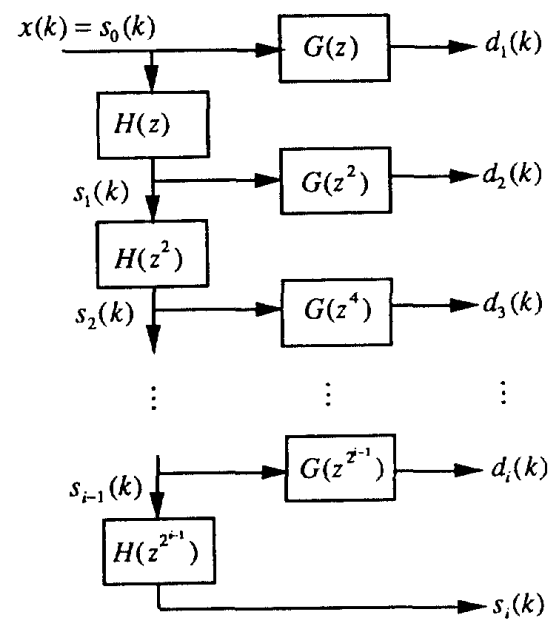

Fig. 1 : Fast iterative implementation of the discrete wavelet frame decomposition. 
It is possible to define an orthogonal DWT in $l_{2}$ by considering the basis functions generated by translating the impulse responses of this filterbank with a step size $2^{i}$ [7]. Such a DWT could, in principle, be obtained by decimating the outputs of the filterbank in Fig. 1 by a factor $2^{i}$, although there is a much more efficient algorithm that essentially uses the same filters $h$ and $g$ over and over again $[1,7]$.

\subsection{Wavelet frames of $l_{2}$}

A simple integer shift of the input signal will usually result in a non-trivial modification of the DWT, as it is usually defined. As far as feature extraction is concerned, this behavior is inadequate since one usually thinks of "texture" as a translation-invariant (or stationary) property. A natural way to overcome this limitation is to perform an analysis in terms of the family of functions $S=\left\{g_{1}(k-l), \cdots, g_{l}(k-l), h_{l}(k-l)\right\}_{l \in Z}$, which is precisely what is done with the algorithm in Fig. 1. This decomposition has a number a remarkable properties which are associated with the mathematical concept of a frame. These include energy conservation and a particularly simple reconstruction algorithm.

Proposition 1 : The family of sequences $S$ is a tight frame of the Hilbert space $l_{2}$.

A proof of this result is given in [8].

\subsection{Texture characterization}

To extend these approaches to higher dimensions, we use a standard tensor product formulation. In two dimensions, there will be four distinct types of basis functions (or filters) corresponding to the different cross-products of the $1 D$ functions $h_{i}$ and $g_{i}$.

The filter bank analysis system shown in Fig. 1 can be viewed as a special case of the local linear transform method [6]. In this approach, we rearrange the output of the filter bank into the $N$-component vector and interpret the result of the analysis for a given spatial index $(k, l)$ as a local linear transformation of the input vector $\mathrm{x}(k, l)$; the latter is just a block representation of the input image centered on the current position. The texture is then characterized by the set of $N$ first order probability density functions. Alternatively, we can get a more compact representation in terms of the channel variances. A statistical justification for this approach can be found in [6]. The success of this method obviously depends on the judicious selection of the filter bank.

In practice, the channel variances are estimated from the average sum of squares over a region of interest $R$ of the given texture type.

\section{TEXTURE CLASSIFICATION}

Classification experiments were conducted using 12 standard 256x256 Brodatz textures. The images had their histogram equalized with a requantization to 32 levels making them indistinguishable on the basis of first order statistics only. The wavelet and filter bank decompositions were performed by processing the individual images globally. For each texture, a total of 64 independent feature vectors (channel variances) was evaluated over a series of $32 \times 32$ non-overlapping sub-regions. The class conditional probability density functions were assumed to be multivariate Gaussian and the subimages were classified using a minimum enror Bayes classifier. For each pattern tested, the training was performed on the remaining set ("Leaving one-out" method) using the maximum likelihood estimates of the distribution parameters.

\begin{tabular}{lccc}
\hline Type of decomposition & $n=0$ & $n=1$ & $n=3$ \\
\hline $\begin{array}{l}1 \text { scale (4 features) } \\
\text { DWT }\end{array}$ & $91.93 \%$ & $91.80 \%$ & $86.72 \%$ \\
DWF & $96.48 \%$ & $93.88 \%$ & $91.54 \%$ \\
\hline 2 scales (7 features) & & & \\
DWT & $95.44 \%$ & $98.18 \%$ & $97.92 \%$ \\
DWF & $98.83 \%$ & $98.70 \%$ & $98.44 \%$ \\
\hline 3 scales (10 features) & & & \\
DWT & $96.35 \%$ & $98.44 \%$ & $98.31 \%$ \\
DWF & $99.35 \%$ & $99.22 \%$ & $99.22 \%$ \\
\hline
\end{tabular}

Table 1 : Percent of correct classification for various orthogonal discrete wavelet transforms (DWT) and wavelet frames (DWF) as a function of the order $\boldsymbol{n}$ and the number of scaies $I$.

We chose to compare the performance of the discrete wavelet transform (DWT) and wavelet frame (DWF) approaches. For this purpose, we considered the series of quadrature mirror filters associated with the Battle-Lemarié orthogonal spline wavelet transforms $[1,4]$.

The classification results for splines of degree $n=0,1$, and 3 are given in Table I. The first observation is that the DWF method always outperforms the DWT, which is consistent with our expectation. In some cases, the improvement is quite substantial. It is also clear that a true multiresolution feature extraction with 2 or 3 levels is preferable to a local analysis with one level only. In fact, the DWF with $n=0$ and $l=1$ is equivalent to the local linear transform (LLT) method using the $2 \times 2$ Hadamard transform described in [6].

Other non-orthogonal spline wavelet transforms were also considered [4]. Additional classification experiments and comparisons are reported in [8].

\section{TEXTURE SEGMENTATION}

The DWF approach to feature extraction can also be integrated into a simple multi-component texture segmentation algorithm that is very similar to the system developed in [9]. A detailed description of this procedure together with some experimental results can be found in [8]. The results obtained are quite encouraging.

\section{References}

[1] S.G. Mallat, "A theory of multiresolution signal decomposition: the wavelet representation", IEEE Trans. Pattern Anal. Machine Intell., vol. PAMI-11, pp. 674-693, 1989.

[2] T. Chang and C.-C.J. Kuo, "Texture analysis and classification with tree-structured wavelet transform", USC-SIPI Report No. 198, University of Southern California, 1992.

[3] A. Laine and J. Fan, "Texture classification by wavelet packet signatures", Preprint, University of Florida, 1992.

[4] M. Unser, A. Aldroubi and M. Eden, "A family of polynomial spline wavelet transforms", Signal Processing, vol. 30, pp. 141-162, January 1993.

[5] K.I. Laws, "Textured image segmentation", Ph. D. Thesis, Rept. 940, Image Processing Institute, University of Southern California, January, 1980.

[6] M. Unser, "Local linear transforms for texture measurements", Signal Processing, vol. 11, pp. 61-79, July 1986.

[7] O. Rioul, "A discrete-time multiresolution theory", IEEE Trans. Signal Proccessing, to appear.

[8] M. Unser, "Texture classification and segmentation using wavelets", NCRR Report 149/92, National Institutes of Health, 1992.

[9] M. Unser and M. Eden, "Multi-resolution feature extraction and selection for texture segmentation", IEEE Trans. Paltern Anal. Mach. Intell., vol. PAMI-11, pp. 717-728, July 1989. 